\title{
Uso de algoritmo de otimização na determinação de tipos de animal funcional
}

\author{
Use of optimization algorithm for determining of animal functional types
}

\author{
Carlos Eduardo Nogueira Martins ${ }^{\mathrm{I}}$ Sérgio Augusto Ferreira de Quadros ${ }^{\mathrm{II}}$ \\ José Pedro Pereira Trindade ${ }^{\mathrm{III}}$ Fernando Luiz Ferreira de Quadros ${ }^{\mathrm{IV}}$
}

\section{RESUMO}

O presente trabalho teve como objetivo avaliar o uso de algoritmo de otimização na determinação de tipos funcionais de animais da raça Braford para diferentes condições ambientais. Foram utilizados algoritmos específicos de otimização por meio do progama computacional SYNCSA, com o objetivo de determinar subconjuntos ótimos de atributos que definem diferentes tipos funcionais, tendo em vista a eficiência de utilização da pastagem pelos animais. Tais algoritmos estão fundamentados na definição de matrizes de incidência que relacionam as variáveis de interesse. No presente estudo, a matriz $B$ definiu a relação animais $x$ medidas corporais; a matriz $W$, a relação produtividade acumulada $x$ graus de sangue; e a matriz $E$, a relação graus de sangue $x$ ambientes (com e sem restrição alimentar). Os resultados mostraram a existência de três tipos funcionais: animais de maior estrutura corporal; animais de estrutura intermediária e animais com menor estrutura, com exceção do atributo largura do peito. Os animais de maior estrutura corporal obtiveram 30 e 47,7kg a mais de terneiros desmamados, no ambiente sem restrição alimentar, que os animais de estrutura corporal média e pequena, respectivamente. No ambiente com restrição alimentar, o tipo de animal de estrutura média apresentou melhor desempenho produtivo, sendo este 18,5 e $36,8 \%$ superior aos tipos de maior e menor estrutura corporal, respectivamente. Os resultados deste trabalho demonstram que o algoritmo criado para determinação de tipos funcionais pode ser utilizado na busca do melhor biótipo animal para os diferentes sistemas de produção.

Palavras-chave: ambiência, biótipo, interação animalambiente, nível alimentar.

\begin{abstract}
This study aimed to evaluate the use of optimization algorithm to determine functional types of animals Braford for different environmental conditions. We used specific optimization algorithms through the computer program SYNCSA in order to determine optimal subsets of attributes that define different functional types in view the efficiency of grazing by animals. These algorithms are based on the definition of incidence matrices that relate the variables of interest. In this study the matrix $B$ defined the relationship animals $x$ body measurements; the matrix $W$ the relationship accumulated productivity $x$ blood degree; and the matrix $E$ the relationship blood degree $x$ environments (with and without food restriction). These results showed the existence of three functional types: animals with larger body structure, intermediate structure and animals with less structure, except the trait width of the chest. The higher body structure animals had more 30 and $47.7 \mathrm{~kg}$ of weaned calves, in the environment without food restriction, than animals of medium and small body frame, respectively. In the environment with dietary restriction, animals with intermediate body structure productive performed 18.5 and $36.8 \%$ better than higher and lower body structure types, respectively. These results demonstrate that the algorithm designed for determination of functional types be used in search of better animal biotype for different production systems.
\end{abstract}

Key words: ambience, animal-environment interaction, biotype, feed level.

\section{INTRODUÇÃO}

A escolha do tipo de estrutura corporal de bovinos de corte, historicamente, foi determinada ora

\footnotetext{
Instituto Federal Catarinense (IFC), Campus Araquari, BR 280, Km 27, CP 21, 89245-000, Araquari, SC, Brasil. E-mail: carlos.martins@ifc-araquari.edu.br. Autor para correspondência.

IIDepartamento de Zootecnia e Desenvolvimento Rural, Universidade Federal de Santa Catarina (UFSC), Florianópolis, SC, Brasil. IIEmpresa Brasileira de Pesquisa Agropecuária, Embrapa Pecuária Sul (CPPSul), RS, Brasil.

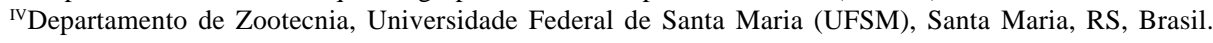


por modismos de raça, ora por exigência dos sistemas de produção (HORIMOTO et al., 2006). Tem-se dado atenção ao estudo do tamanho corporal adequado para bovinos de corte, devido aos requisitos de produção e mantença que, em última análise, influenciam o grau de maturidade fisiológica e o retorno econômico do negócio (ROCHA et al., 2003). Segundo KLOSTERMAN(1972) e FITZHUGH(1978), o tamanho corporal pode apresentar vantagens biológicas importantes quanto aos aspectos relacionados à adaptação, resistência e tipo de exploração, sendo, no entanto, difícil estabelecer o tamanho ideal para todas as situações de exploração.

A utilização de biótipos adaptados a cada sistema de produção, que denominamos como tipos funcionais de animais (TFAs), produz uma relação custo/benefício economicamente positiva. Estudos relatam um efeito negativo do aumento do tamanho corporal sobre o desempenho reprodutivo de vacas, afetando idade à puberdade, período de gestação (VARGAS et al., 1999; MARSON et al., 2001), taxa de concepção, taxa de parição (BUTTRAM et al., 1989), época de parição dentro da estação de monta (VARGAS et al., 1999), peso ao nascer, taxa de sobrevivência de bezerros de novilhas e taxa de desmama (OLSON et al., 2002; VARGAS et al., 1999).

A existência de uma relação direta entre o tamanho corporal e eficiência de produção em bovinos de corte tem levado à inclusão de características associadas ao tamanho, massa e dimensão dos animais em programas de seleção (ARANGO \& PLASSE, 2002). Os programas de melhoramento genético implementados nas diversas raças têm enfocado, no seu delineamento, principalmente, a determinação do objetivo de seleção e dos critérios de seleção objetivo, observando a facilidade de mensuração, a variabilidade genética e a importância econômica (SILVA et al., 2004).

HAGGER \& HOFER (1989) destacaram que as medidas corporais são interessantes, pois são fáceis de obter. Segundo WALTERS \& FRY (2003), a medida de diferentes partes do corpo permite ao produtor reconhecer defeitos estruturais e funcionais que são genéticos, e possíveis problemas que surjam de fenótipo impróprio.

O uso de tipos funcionais tem sido amplamente adotado nos estudos das comunidades vegetais em que algoritmos de otimização, como o proposto por PILLAR \& SOSINSKI (2003), auxiliam na determinação dos tipos funcionais de plantas, os quais são caracterizados por grupos de plantas que exibem respostas similares às condições de ambiente e apresentam efeitos parecidos sobre os processos dominantes do ecossistema (PILLAR \& ORLÓCI, 1993).
A lógica desses algoritmos de busca utilizados para comunidades vegetais permite-nos considerar a sua aplicação na tipificação de animais.

Nesse sentido, o presente trabalho teve como objetivo avaliar o uso de algoritmo de otimização na determinação de tipos funcionais de animais para diferentes condições ambientais.

\section{MATERIAL E MÉTODOS}

O trabalho foi realizado na Fazenda Meia Lua, localizada no município de Itapema, litoral do Estado de Santa Catarina, Brasil. O relevo apresenta topografia plana e suavemente ondulada, com solos siltosos-argilosos com manchas arenosas e presença de turfa úmida, tendo, em alguns pontos, mais de 10\% de matéria orgânica e altitude média de 3 metros. O clima da região é Cfa - subtropical úmido, segundo a classificação climática de Köeppen, com índice pluviométrico de 2000-2800mm ano ${ }^{-1}$ e temperatura média anual de $19^{\circ} \mathrm{C}$. Os animais são alimentados exclusivamente a pasto, em pastagens onde predominam Brachiaria decumbens e B. arecta, sendo suplementados com mistura mineral, caracterizando um ambiente sem restrição alimentar (ASRA). O sistema de pastoreio utilizado é o rotativo com tempo de permanência dos animais nos piquetes de quatro a cinco dias. $\mathrm{O}$ desmame aconteceu entre sete e oito meses de idade e o critério para a entrada das novilhas na vida reprodutiva foi o peso de $285 \mathrm{~kg}$. As vacas que desmamam terneiros com peso abaixo da média anual do rebanho são realocadas em piquetes de pastagem natural, onde predominam o capim-limão (Cymbopogon citratus), samambaia (Pteridium aquilinum) e espécies da família das ciperáceas, caracterizando um ambiente com restrição alimentar(ACRA).

Nos dias 07 e 14 de julho de 2005, foram realizadas as mensurações corporais em 80 vacas pertencentes a diferentes graus de sangue Nelore $\mathrm{x}$ Hereford, com idade média de sete anos. Deste total de animais, 59 (9 1/2Nelore x 1/2Hereford; 2 3/4Nelore $x$ 1/4 Hereford; 22 1/4Nelore x 3/4 Hereford; 26 3/8Nelore $x$ 5/8Hereford) pertenciam ao ASRA e 21 ( 7 1/2Nelore $x$ $1 / 2$ Hereford; 11 3/4Nelore x $1 / 4$ Hereford; $21 / 4$ Nelore $\mathrm{x}$ 3/4Hereford; $13 / 8$ Nelore $x$ 5/8Hereford) ao ACRA. Durante as avaliações, tomou-se o devido cuidado para posicionar corretamente os animais, com a cabeça erguida e com os quatro membros apoiados perpendicularmente ao solo. Utilizou-se uma régua para obtenção das medidas da altura das cruzes (AC), altura da garupa (AG) e largura do peito (LP), um paquímetro para largura da garupa (LG) e uma fita métrica para os comprimentos do pescoço (CP), dorso (CD), garupa (CG), corpo (CC) e as circunferências torácica (CT) e do flanco (CF). 
A AC foi obtida pela diferença entre a altura total do brete e a distância perpendicular entre a extremidade superior deste e as cruzes. Procedimento semelhante foi adotado para a obtenção da AG, neste caso, o limite inferior era o dorso do animal na linha do ílio. A largura do peito foi obtida descontando da largura do brete a soma dos valores obtidos por duas pequenas réguas que, partindo das laterais do brete, mediam a distância até atingir as articulações escápulo-humerais. A distância entre a saliência dos ílios correspondeu a LG. A medida CC foi tomada lateralmente entre a extremidade ventral da espádua e a ponta do ísquio, o CP, da articulação da primeira vértebra cervical com o osso occipital até a linha das bordas dorsais da escápula, o CD, da linha das bordas dorsais da escápula até a linha dos ílios, o CG, da linha dos ílios até a inserção da cauda. A medida do comprimento do tronco (CTr) foi obtida pelo somatório do CP, CD e CG. Amedida CT foi tomada pelo contorno do tórax, passando pelo cilhadouro e voltando perpendicularmente à linha do dorso e a CF pela circunferência do abdômen na região do ílio.

O parâmetro utilizado para expressar o desempenho produtivo das vacas foi o índice de produtividade acumulada (PAC), que indica a produtividade da fêmea em quilogramas de terneiros desmamados por ano. Este índice mede a capacidade da vaca em se reproduzir regularmente, a uma menor idade e desmamar animais com maior peso (LÔBO et al., 2000). A PAC é obtida pela fórmula: $\mathrm{PAC}=\left(\mathrm{P}_{\mathrm{d}} \cdot \mathrm{n}_{\mathrm{p}} \cdot \mathrm{C}_{\mathrm{a}}\right) /\left(\mathrm{IVP}_{\mathrm{n}}-\mathrm{C}_{\mathrm{i}}\right)$, em que: $P_{d}=$ média do peso dos bezerros à desmama, $n_{p}=$ número total de bezerros produzidos, $c_{a}=$ constante igual a 365 dias, que permite expressar a fertilidade em base anual, $I V P_{n}=$ idade da vaca por ocasião do último parto, $c_{i}=$ constante igual a 550 dias, aproximadamente 18 meses, tendo-se em vista a expectativa do primeiro parto ocorrer ao redor de 30 meses de idade.

No programa computacional SYNCSA (PILLAR, 2000), foram executados algoritmos de otimização para determinação de subconjuntos ótimos de atributos que definem tipos funcionais, nos quais a descrição das populações (animais), por meio dos atributos (medidas corporais), definiu uma matriz B (animais x medidas corporais) e a descrição das comunidades (grau de sangue) pela produtividade acumulada desses animais resultou em uma matriz W (PAC dos animais $\mathrm{x}$ grau de sangue). Uma terceira matriz E descreveu os graus de sangue pelos ambientes, com e sem restrição alimentar. Essas matrizes foram analisadas procurando-se um subconjunto ótimo de atributos que maximizasse a correlação entre a variação dos animais descrita pelos TF definidos com base nesses atributos e a eficiência de utilização da vegetação pelos animais. A medida de correlação utilizada pelo algoritmo de otimização foi a medida de congruência, que representa a correlação de Pearson calculado entre a matriz que contém as dissimilaridades (distância Euclidiana) entre as comunidades descritas pelos TF e a matriz que contém as dissimilaridades (diferenças em valores absolutos) entre os fatores ambientais (PILLAR \& SOSINSKI, 2003). A cada iteração recursiva, a análise de agrupamento UPGMA baseada no índice de similaridade de Gower (ver PODANI, 2000) esteve envolvida como passo adicional na classificação politética, baseada na matriz de populações por atributos. Escolhendo o número de TF (nível de partição dos grupos) com base na máxima congruência, o algoritmo busca, adicionalmente, para cada subconjunto de atributos, um nível de partição que maximize a função objetiva (PILLAR \& SOSINSKI, 2003), que, neste trabalho, foi representada pela PAC nos diferentes ambientes, com e sem restrição alimentar.

O teste de comparação de médias dos TFAs foi realizado via aleatorização com auxílio do programa computacional MULTIV (PILLAR, 2001), cujo objetivo foi testar a hipótese de diferença de PAC dos TFAs nos dois ambientes alimentares.

\section{RESULTADOS E DISCUSSÃO}

A análise dos dados a partir do algoritmo de otimização permitiu a formação de um subconjunto ótimo constituído de dois atributos (comprimento do corpo e circunferência do flanco), os quais apresentaram congruência máxima de 0,38 com a variável ambiental, definindo 15 tipos funcionais (Tabela 1 ).

Na figura 1A, estão representadas as medidas corporais dos animais no espaço de ordenação quando submetidas aos TFAs, em que os eixos I e II sintetizaram $73,7 \%$ da variação total dos dados. Observa-se que os 15 tipos funcionais poderiam ser agrupados em três, os quais estão destacados pelas elipses (Figura 1A). Essa tendência se confirma com o resultado apresentado na tabela 1 , em que um segundo subconjunto ótimo definido por quatro atributos, largura de peito, comprimento, altura e largura de garupa, definiu três tipos funcionais com 0,37 de congruência máxima com a variável ambiental. Esses resultados demonstram a capacidade do algoritmo em sintetizar de forma simples e clara um grande conjunto de dados, permitindo que se possa visualizar diferentes possibilidades de hipóteses.

A análise de ordenação envolvendo os três tipos funcionais conseguiu sintetizar 100\% da variação total dos dados nos dois eixos principais (Figura 1B). Nota-se que o TFA1 teve correlação positiva com todas as medidas corporais, o TFA2 apresentou correlação 
Tabela 1 - Subconjuntos e número de tipos funcionais de animais (TFAs) com máxima congruência.

\begin{tabular}{|c|c|c|c|c|c|c|c|c|c|c|c|c|c|}
\hline Grupos & Congruência & -- & 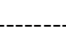 & - &.- & ---Sub & ijunto & $\bmod$ & ibuto & $\ldots$ & $\ldots$ & $\ldots$ & 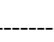 \\
\hline 2 & 0,357 & LP & & & & & & & & & & & \\
\hline 15 & 0,383 & CC & CF & & & & & & & & & & \\
\hline 8 & 0,374 & AG & CT & CF & & & & & & & & & \\
\hline 3 & 0,375 & CG & AG & LP & LG & & & & & & & & \\
\hline 3 & 0,366 & CP & CG & LP & CT & CF & & & & & & & \\
\hline 4 & 0,373 & CD & CG & $\mathrm{CTr}$ & AG & LP & CF & & & & & & \\
\hline 3 & 0,360 & $\mathrm{CP}$ & $\mathrm{CTr}$ & AG & $\mathrm{AC}$ & LP & LG & CT & & & & & \\
\hline 4 & 0,357 & $\mathrm{CP}$ & $\mathrm{CTr}$ & $\mathrm{CC}$ & AG & $\mathrm{AC}$ & LP & LG & CT & & & & \\
\hline 3 & 0,365 & CP & CG & $\mathrm{CTr}$ & CC & AC & LP & LG & CT & CF & & & \\
\hline 2 & 0,340 & EC & $\mathrm{CP}$ & $\mathrm{CD}$ & CG & $\mathrm{CTr}$ & $\mathrm{CC}$ & AG & LP & LG & $\mathrm{CF}$ & & \\
\hline 2 & 0,325 & $\mathrm{CP}$ & $\mathrm{CD}$ & CG & $\mathrm{CTr}$ & CC & AG & AC & LP & LG & $\mathrm{CT}$ & CF & \\
\hline 2 & 0,246 & EC & $\mathrm{CP}$ & CD & CG & $\mathrm{CTr}$ & CC & AG & AC & LP & LG & CT & CF \\
\hline
\end{tabular}

CP: Comprimento Pescoço; CD: Comprimento Dorso; CG: Comprimento Garupa; CTr: Comprimento Tronco; CC: Comprimento Corpo; AG: Altura Garupa; AC: Altura Cruzes; LP: Largura Peito; LG: Largura Garupa; CT: Circunferência Torácica; CF: Circunferência Flanco; EC: Escore Corporal.

negativa com as medidas corporais, com exceção da largura do peito, e o TFA3 apresentou comportamento inverso ao do TFA2 (Figura 1B). Dessa forma, o TFA1 pode ser caracterizado como o grupo de animais de maior estrutura corporal, o TFA3, por animais de estrutura intermediária e os do TFA2, por animais com menor estrutura, com exceção da largura do peito (Tabela 2).

Apesar de não ter sido encontrada diferença significativa $(\mathrm{P}<0,05)$ entre a PAC dos TFAs nos dois ambientes, observa-se que o TFA1 (maior estrutura corporal) no ASRA obteve 30 e $47,7 \mathrm{~kg}$ a mais de terneiros desmamados que os TFAs de estrutura corporal média e pequena, respectivamente (Tabela 2). A melhor PAC dos animais de maior estrutura corporal pode estar associada à maior capacidade de produção de leite e, conforme ESTEVES (1999), vacas de garupas mais largas tendem a apresentar maior facilidade de partos e indiretamente contribuem para uma melhor eficiência reprodutiva, pois um parto distócico causa vários outros problemas como retenção de placenta, prolapso uterino, debilidade, ou seja, fatores que contribuem para o aumento do intervalo entre partos.

Já no ACRA, o TFA de estrutura média apresentou melhor desempenho, sendo este 18,5 e $36,8 \%$ superior aos TFAs de maior e menor estrutura corporal, respectivamente. Este resultado deve-se ao fato de, geralmente, vacas maiores apresentarem maior perda de peso em condições adversas, o que resulta em intervalos de partos mais longos (FITZHUGH, 1978). Nesse caso, o estresse alimentar não foi tão severo a ponto de limitar a produtividade dos animais de estrutura corporal média. Apesar do baixo número de unidades amostrais neste grupo (Tabela 2), existe tendência de que, para manter uma produção razoável em ambientes/anos ruins, os animais de estrutura intermediária seriam mais apropriados, sendo não interessante manter TFAs de estrutura corporal pequena neste rebanho, pois, mesmo no ambiente com restrição alimentar, estes apresentaram desempenho inferior.

BARCELLOS et al. (2001), ao avaliarem os efeitos da estrutura corporal (altura da garupa) sobre a idade à puberdade de novilhas Braford, distribuídas em três grupos, conforme a altura da garupa no desmame (7 meses): pequenas (97 a 109cm), médias (110 a $115 \mathrm{~cm})$ e grandes (116 a $122 \mathrm{~cm})$, observaram que as novilhas de maior estrutura corporal foram mais pesadas na puberdade $(369,2 \mathrm{~kg})$ do que as dos grupos pequenas $(323,3 \mathrm{~kg})$ e médias $(337,1 \mathrm{~kg})$. Entretanto, foram 56 e 36 dias mais tardias na idade de aparecimento da puberdade do que as novilhas dos grupos pequenas e médias, respectivamente.

Cabe ressaltar que, mesmo em ambientes onde vacas de maior porte apresentam melhor desempenho por unidade animal, isso pode não ser verdadeiro, quando o parâmetro for produção por unidade de área. Deve-se avaliar a conveniência de se utilizar este tipo de animal maior em termos de carga animal e de produção por unidade de superfície, pois, segundo DI MARCO et al. (2006), as vacas de menor tamanho produzem mais quilos de terneiros por unidade de superfície, mesmo que o peso ao desmame das progênies desses animais seja inferior. Entretanto, segundo estes autores, isso não indica que estes animais sejam mais eficientes. Ao se abrir mão do 


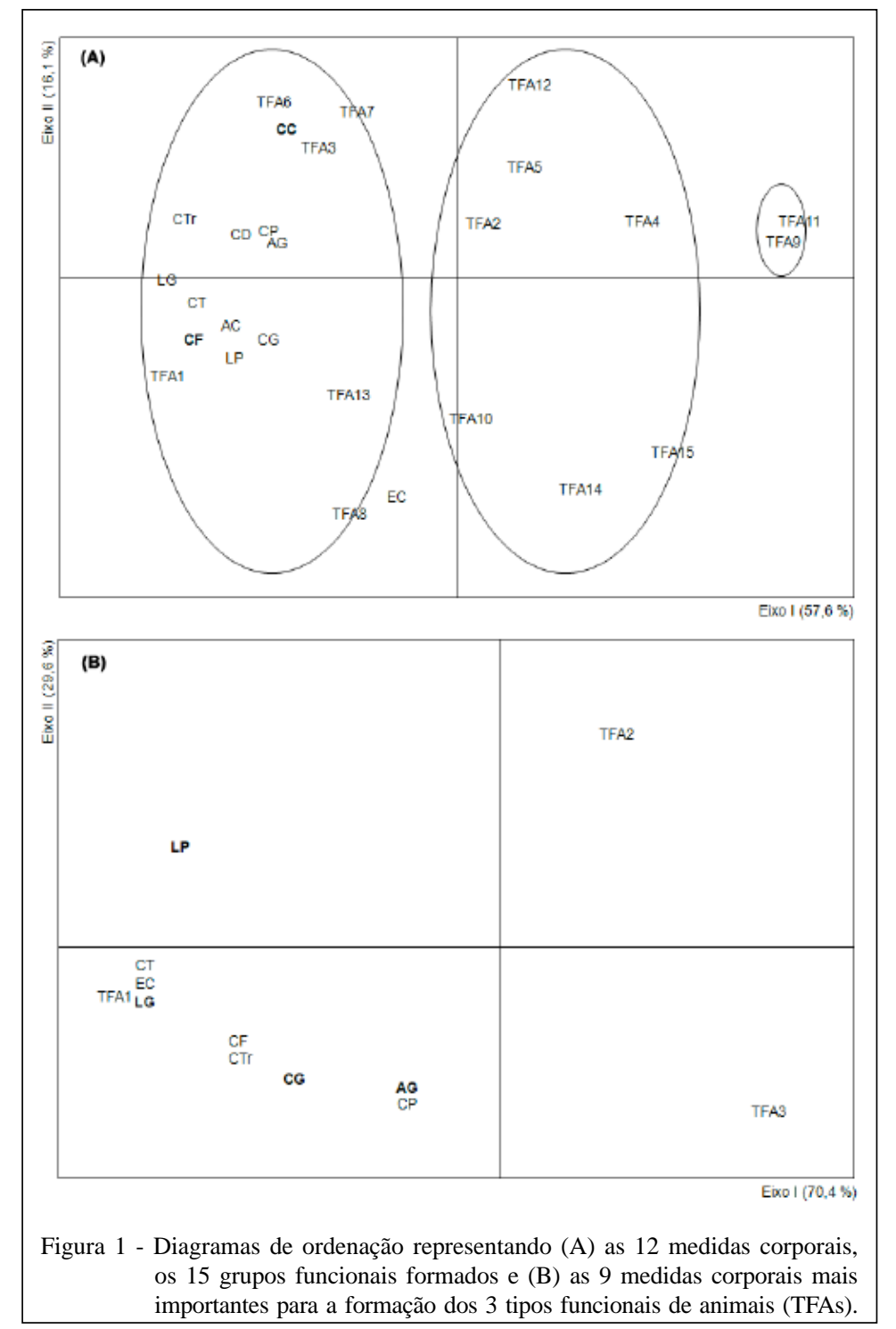

número de ventres por área, deve-se tentar um preço maior ou uma maior facilidade de colocação dos terneiros produzidos para compensar esta redução no número de vacas no sistema.

CHRISTOFARI et al. (2008), ao caracterizar os efeitos dos aspectos dependentes da genética de bezerros comercializados no estado do Rio Grande do Sul e verificar sua influência no preço de venda, observaram que, para características de tamanho e desenvolvimento muscular, os bezerros grandes e fortes tiveram maior liquidez em comparação aos de estrutura média ou pequena, portanto, têm maior preço e valorização por kg de peso vivo.

Destaca-se que, para cada raça e ambiente, existe um biótipo ideal, o qual permitirá o maior retorno econômico ao produtor. Conforme DI MARCO et al. (2006), existem importantes diferenças entre animais de tamanho estrutural diferente quanto ao peso de abate ótimo, grau de terminação, produtividade e eficiência, que são importantes para determinar que tipo de animal melhor se adapta a certas condições de produção, já que, com raras exceções, os sistemas de pastoreio têm limitações nutricionais de diferentes durações e intensidades, devido às flutuações da quantidade e qualidade da forragem, durante o ano e entre anos.

Os resultados encontrados na literatura, conjuntamente com os deste trabalho, demonstram que não existe um tipo funcional de animal ideal para todas as situações. Portanto, a identificação de indivíduos cujo biótipo seja mais adaptado às condições locais de produção deve constar nos programas de seleção.

Ciência Rural, v.42, n.8, ago, 2012. 
Tabela 2 - Medidas corporais e produtividade acumulada de acordo com os tipos funcionais de animais (TFAs).

\begin{tabular}{|c|c|c|c|c|c|c|c|c|c|c|c|c|}
\hline \multirow{2}{*}{ Tipos Funcionais de Animais } & \multirow[b]{2}{*}{$\mathrm{CP}$} & \multirow[b]{2}{*}{ CD } & \multirow[b]{2}{*}{ CG } & \multirow[b]{2}{*}{$\mathrm{CTr}$} & \multirow[b]{2}{*}{ CC } & \multirow[b]{2}{*}{ AG } & \multirow[b]{2}{*}{$\mathrm{AC}$} & \multirow[b]{2}{*}{ LP } & \multirow[b]{2}{*}{ LG } & \multirow[b]{2}{*}{ CT } & \multirow[b]{2}{*}{$\mathrm{CF}$} & \multirow[b]{2}{*}{ EC } \\
\hline & & & & & & & & & & & & \\
\hline TFA1 & 61 & 80 & 30 & 180 & 140 & 130 & 120 & 45 & 52 & 180 & 190 & 3 \\
\hline TFA2 & 59 & 80 & 27 & 160 & 140 & 120 & 120 & 43 & 48 & 170 & 170 & 2 \\
\hline \multirow{3}{*}{ TFA3 } & 61 & 80 & 29 & 170 & 140 & 130 & 120 & 39 & 48 & 170 & 180 & 2 \\
\hline & \multicolumn{12}{|c|}{ 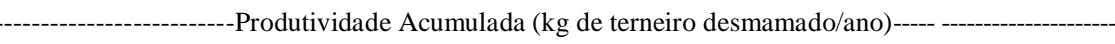 } \\
\hline & \multicolumn{4}{|c|}{ Ambiente } & \multicolumn{4}{|c|}{ Média* } & \multicolumn{4}{|c|}{$\mathrm{n}$} \\
\hline \multirow{2}{*}{ TFA1 } & \multicolumn{4}{|c|}{ Sem Restrição } & \multicolumn{4}{|c|}{$202,0 \mathrm{a}$} & \multicolumn{4}{|c|}{30} \\
\hline & \multicolumn{4}{|c|}{ Com Restrição } & \multicolumn{4}{|c|}{$119,5 b$} & \multicolumn{4}{|c|}{7} \\
\hline \multirow{2}{*}{ TFA2 } & \multicolumn{4}{|c|}{ Sem Restrição } & \multicolumn{4}{|c|}{$154,3 a$} & \multicolumn{4}{|c|}{20} \\
\hline & \multicolumn{4}{|c|}{ Com Restrição } & \multicolumn{4}{|c|}{$103,5 b$} & \multicolumn{4}{|c|}{5} \\
\hline \multirow{2}{*}{ TFA3 } & \multirow{2}{*}{\multicolumn{4}{|c|}{$\begin{array}{l}\text { Sem Restrição } \\
\text { Com Restrição }\end{array}$}} & \multicolumn{4}{|c|}{ 172,0a } & \multicolumn{4}{|c|}{8} \\
\hline & & & & & & & & & & & & \\
\hline
\end{tabular}

* Médias seguidas de letras distintas, dentro de cada tipo funcional, apresentam diferença significativa $(\mathrm{P}<0,05)$.

CP: Comprimento Pescoço; CD: Comprimento Dorso; CG: Comprimento Garupa; CTr: Comprimento Tronco; CC: Comprimento Corpo; AG: Altura Garupa; AC: Altura Cruzes; LP: Largura Peito; LG: Largura Garupa; CT: Circunferência Torácica; CF: Circunferência Flanco; EC: Escore Corporal.

\section{CONCLUSÃO}

Os resultados deste trabalho demonstram que o algoritmo de otimização criado para determinação de tipos funcionais de plantas também pode ser utilizado na busca do melhor biótipo animal para os diferentes sistemas de produção. Entretanto, a utilização dessa técnica como uma ferramenta para auxiliar nas tomadas de decisões na propriedade depende de um controle mais rigoroso dos dados zootécnicos dos animais por parte dos produtores. Sugere-se, como futuros trabalhos, a utilização deste algoritmo de busca com um conjunto de dados mais amplo, como o de associações de raças.

\section{COMITÊ DE ÉTICA E BIOSSEGURANÇA}

Declaramos que o trabalho em questão levou em consideração o bem-estar dos animais durante todo o período experimental.

Nesse sentido, assumimos as responsabilidades do trabalho para possíveis esclarecimentos.

\section{REFERÊNCIAS}

ARANGO, J.; PLASSE, D. Cow weight in a closed Brahman herd. In: WORLD CONGRESS ON GENETICS APPLIED TO LIVESTOCK PRODUCTION, 7., 2002, Montpellier, France. Proceedings... Montpellier: INRA, 2002. CD.

BARCELLOS, J.O.J. et al. Influência da estrutura corporal na idade à puberdade de novilhas Braford. In: REUNIÃO ANUAL DA SOCIEDADE BRASILEIRA DE ZOOTECNIA, 38., 2001,
Piracicaba, SP. Anais... Piracicaba: Fundação de Estudos Agrários Luiz de Queiroz, 2001. p.397-398.

BUTTRAM, S.T.; WILHAM, R.L. Size and management effects on reproduction in first, second and third-parity beef cow. Journal of Animal Science, v.67, p.2191-2196, 1989. Disponível em: <http://jas.fass.org/content/67/9/2191>. Acesso em: 24 mar. 2010.

CHRISTOFARI, L.C. et al. Tendências na comercialização de bezerros relacionadas às características genéticas no Rio Grande do Sul. Revista Brasileira de Zootecnia, v.37, n.1, p.171176, 2008. Disponível em: <http://dx.doi.org/10.1590/S151635982008000100025>. Acesso em: 26 out. 2010. doi: 10.1590/S1516-35982008000100025.

DI MARCO, O.N. et al. Crescimento de bovinos de corte. Porto Alegre: Departamento de Zootecnia da Universidade Federal do Rio Grande do Sul, 2006. 248p.

ESTEVES, A.M.C. Características lineares e suas relações com aspectos reprodutivos em rebanhos leiteiros. Revista Brasileira de Reprodução Animal, v.23, n.1, p.16-19, 1999.

FITZHUGH, H.A. Animal size and efficiency with special reference to the breeding female. Animal Production, v.27, p.393-401, 1978.

HAGGER, C.; HOFER, A. Phenotypic and genetic relationships between wither height, heart girth and milk yield in Swiss Braunvieh and Simmental breeds. Livestock Production Science, v.28, p.265-271, 1991. Disponível em: <http://dx.doi.org/10.1016/ 0301-6226(91)90147-I>. Acesso em: 17 maio, 2010. doi: http:/ /dx.doi.org/10.1016/0301-6226(91)90147-I.

HORIMOTO, A.R.V.R. et al. Estimation of genetic parameters for a new model for defining body structure scores (frame scores) 
in Nellore cattle. Genetics and Molecular Research, v.5, p.828-836, 2006. Disponível em: <http://www.geneticsmr.com/ /year2006/vol5-4/pdf/gmr0200.pdf>. Acesso em: 08 ago. 2009.

KLOSTERMAN, E.W. Beef cattle size for maximum efficiency. Journal of Animal Science, v.34, p.875-880, 1972. Disponível em: <http://jas.fass.org/content/34/5/875>. Acesso em: 15 abr. 2010.

LÔBO, R.B. et al. Avaliação genética de animais jovens, touros e matrizes. Ribeirão Preto: Universidade de São Paulo/ GEMAC, 2000. 90p.

MARSON, E.P.; FERRAZ, J.B.S. Fatores genéticos relacionados à idade à puberdade em novilhas de corte. São Paulo. Disponível em: <http://www.beefpoint.com.br/bn/ radarestecnicos/artigo.asp? area $=14 \&$ area_desc+ Melhoramento+Gen\%E9tico\&id_artigo=1747\&perM=5\&perA=2003>. Acesso em: 15 jun. 2004.

OLSON, T.A. et al. A relação entre frame size e características de fertilidade em gado Brahman. Disponível em: <http://www.abcz.com.br>. Acesso em: 23 jun. 2002.

PILLAR, V.D. SYNCSA: software for character-based community analysis. Porto Alegre: UFRGS, 2000. Disponível em: <http://ecoqua.ecologia.ufrgs.br/arquivos/Software>. Acesso em: 14 set. 2009.

PILLAR, V.D. MULTIV: aplicativo para análise multivariada e teste de hipóteses. Porto Alegre: UFRGS, 2001. CD.

PILLAR, V.D.; ORLÓCI, L. Character-based community analysis; the theory and an application program. The Hague, SPB Academic Publishing, 1993. 270p.
PILLAR, V.D.; SOSINSKI, E.E. An improved method for searching plant functional types by numerical analysis. Journal of Vegetation Science, v.14, p.323-332, 2003. Disponível em: <http://onlinelibrary.wiley.com/doi/10.1111/j.16541103.2003.tb02158.x/pdf>. Acesso em: 18 jul. 2009. doi: 10.1111/j.1654-1103.2003.tb02158.x.

PODANI, J. Introduction to the exploration of multivariate biological data. Leiden: Backhuys Publishers, 2000. 407p.

ROCHA, E.D. et al. Mature Nellore cow size and its effect on beef cattle production system. Arquivo Brasileiro de Medicina Veterinária e Zootecnia, v.55, n.4, p.474-479, 2003. Disponível em: <http://dx.doi.org/10.1590/S0102$09352003000400014 \mathrm{f}>$. Acesso em: 11 jun. 2010. doi: 10.1590/S0102-09352003000400014.

SILVA, J.A. II de V. et al. Programa de melhoramento genético das raças Brangus e Braford. In: SIMPÓSIO DA SOCIEDADE BRASILEIRA DE MELHORAMENTO ANIMAL, 5., 2004, Pirassununga, SP. Anais... Pirassununga, SP: Sociedade Brasileira de Melhoramento Animal, 2004. CD.

VARGAS, C.A. at al. Influence of frame size and body condition score on performance of Brahman cattle. Journal of Animal Science, v.77, p.3140-3149, 1999. Disponível em: <http:// jas.fass.org/content/77/12/3140>. Acesso em: 05 abr. 2010.

WALTERS, C.; FRY, G. Reproduction and animal health. Austin, TX: ACRES, 2003. 218p. 